-Editorial·

\title{
Selective role of autophagy in neuronal function and neurodegenerative diseases
}

\author{
Yan-Ning Rui ${ }^{1}$, Weidong Le ${ }^{2,3, *}$ \\ ${ }^{1}$ The University of Texas Health Science Center at Houston, Texas, USA \\ ${ }^{2}$ Center for Translational Research on Neurological Diseases, First Affiliated Hospital, Dalian Medical University, Dalian \\ 116011, China \\ ${ }^{3}$ Institute of Health Science, Shanghai Institutes for Biological Science, Chinese Academy of Science, Shanghai 200025, \\ China
}

${ }^{*}$ Corresponding author and Guest Editor of the Special Issue. E-mail: wdle@sibs.ac.cn

(C) Shanghai Institutes for Biological Sciences, CAS and Springer-Verlag Berlin Heidelberg 2015

Proteostasis is critical for neuronal maintenance and survival, and its imbalance leads to neurodegeneration with the hallmark of protein misfolding and aggregation ${ }^{[1]}$. Macroautophagy becomes a major route for the clearance of protein aggregates that are normally poor substrates for the proteasome, the other protein quality-control machinery ${ }^{[2]}$. As a flux process, macroautophagy (hereafter referred to as autophagy) involves the formation of the autophagosome, a double-membrane vesicle for engulfing unwanted cellular components such as protein aggregates, and the fusion of autophagosomes with lysosomes that contain many potent proteases for final degradation ${ }^{[3]}$.

In the past decades, the mechanism of autophagy has been intensively studied under starvation conditions. Autophagy is a hierarchical process involving the activation of ULK1, the initiating kinase, and many downstream events such as Beclin 1 complex activation and LC3 lipidation ${ }^{[3]}$. It has been well established that, during amino-acid starvation, mTOR, the master nutrient sensor, dissociates from and in turn activates ULK1 kinase ${ }^{[4]}$. Interestingly, upon energy starvation such as glucose depletion, the main energy sensor AMPK activates ULK1 through antagonizing mTOR-mediated ULK1 inhibitory phosphorylation $^{[5]}$. It is generally believed that starvation induces non-selective autophagy that targets non-essential cellular components for degradation to provide building blocks for cellular survival in unfavorable conditions.

In comparison with the well-characterized starvation- induced nonselective autophagy, the molecular mechanism of selective autophagy is largely unknown ${ }^{[6]}$. Selective autophagy is a comprehensive term that can be classified into many different types such as aggrephagy, mitophagy, and lipophagy ${ }^{[7]}$. In particular, aggrephagy plays an important role in neuronal survival, as neurons are nondividing cells and cannot overcome cellular toxicity induced by protein aggregates through dilution effects. Therefore, aggrephagy becomes essential for neurons to preserve proteostasis. However, the underlying mechanism is yet to be uncovered. Recently, Huntingtin, the Huntington's disease gene product, was identified as a scaffold protein for selective autophagy including aggrephagy, mitophagy, and lipophagy in mammalian cells, paving the way for further investigation in this emerging young field ${ }^{[8]}$. In addition, cargo receptor proteins such as p62 and NBR1 bring cargos to autophagosomes by interacting with the autophagosomal protein $\mathrm{LC}^{[9]}$. As both p62 and NBR1 contain ubiquitin-binding domains, the cargos modified by lineage-specific ubiquitin play an important role in cargo recognition in selective autophagy. For instance, ubiquitin K63-modified substrates preferentially bind to p62 for autophagic degradation ${ }^{[10]}$.

Selective autophagy has been linked to a variety of human diseases including but not limited to neurodegeneration and cancer ${ }^{[11]}$. For example, tau protein, implicated in Alzheimer's disease (AD), was shown to be an autophagy substrate, and increasing autophagy- 
mediated tau degradation ameliorates disease progression in $A D$ models ${ }^{[12,13]}$. Autophagy dysregulation has also been reported in amyotrophic lateral sclerosis (ALS), another neurodegenerative disease that can be caused by SOD1 mutations, and enhancing autophagy by pharmaceutical or genetic methods can improve the clinical symptoms in animal models of ALS, supporting the notion that autophagy is a promising translational target ${ }^{[14-16]}$.

In this special issue, "Autophagy in Neural Function and Neurodegeneration", we present 13 high-quality, peer-reviewed articles. Among them, 8 are reviews and 5 are original research papers, which cover a broad basic research on autophagy with regard to neuronal function, synaptic development, neuronal cell death and neuroprotection, and clinical relevant research on neurological and psychological disorders (AD, ALS and other motor neuron diseases, depression, multiple sclerosis, Parkinson's disease and stroke) ${ }^{[15,17-28]}$.

Selective autophagy is generally believed to be beneficial to neuronal development and functions ${ }^{[17-19]}$. However, in some contexts, dysregulation of autophagy may cause neurodegeneration and cell death ${ }^{[20,21]}$. Rubinsztein and colleagues ${ }^{[20]}$ have provided an excellent review of this important field by analyzing several key findings on autophagic cell death and they proposed a new model on how the extent of autophagy dictates the fate of neurons under stress, which quite nicely explains why autophagy can play opposing roles in neurons depending on the context. In addition to selective macroautophagy, chaperone-mediate autophagy (CMA), another type of selective autophagy, also plays an important role in neuronal survival, as many aggregate-prone proteins such as $\alpha$-synuclein and tau serve as its targets. The detailed relationship between neuroprotection and CMA is reviewed by Dr. Yang ${ }^{[19]}$. Equally importantly, the members of Dr. Mao's lab fully discuss how to prevent neurodegeneration by targeting the CMA pathway ${ }^{[21]}$. In his review, Dr. Mao outlines the key molecular players in CMA and the stressors associated with it, and analyzed the role of CMA in neurodegeneration, concluding that it is a potential therapeutic target.

It is becoming clearer that autophagy dysregulation plays an important role in several major neurological and psychological disorders ${ }^{[15,22-28]}$. In this special issue,
Dr. Chen's group give an in-depth review on the role of mitophagy in ischemic brain injury ${ }^{[22]}$. They discussed several stressors that cause mitophagy activation including endoplasmic reticulum (ER) stress, oxidative stress, and excitotoxicity, as well as mitophagy mediated by the three pathways PINK1/Parkin, Bnip3/Nix, or FUNDC ${ }^{[22]}$. Liang et al. ${ }^{[23]}$ used meta-analysis to identify a critical autophagy signaling network that contributes to stroke in the ischemic rodent brain. Interestingly, dysfunction of mitophagy and CMA has recently been found to be involved in multiple sclerosis (MS), a permanent neurological impairment typical of chronic inflammatory demyelinating disorders ${ }^{[24]}$. As MS is an auto-immune disease of the central nervous system, the connection between neuro-immunology and autophagy is gaining increasing attention ${ }^{[24]}$. For example, the activation of autophagy may participate in $A \beta$ vaccineinduced $A \beta$ clearance in $A D^{[25]}$.

Non-selective or selective autophagy share the same "trash-can" - the lysosome - which means that if autophagic flux is aberrant, simply boosting the biogenesis of autophagosomes is not enough to solve the problem. For example, an autophagic flux defect in ALS is reported in the original article by Dr. Le's lab ${ }^{[15]}$ and this is ameliorated by overexpression of histone deacetylase 6 , an adaptor protein promoting autophagosome-lysosome fusion. CHIP, an E3 ligase, is also considered to be an important player in regulating autophagic flux associated with ALS and other motor neuron diseases ${ }^{[26]}$. In addition to these neurological diseases, autophagy dysregulation is believed to contribute to the pathophysiology of major depressive disorder and many anti-depressants are autophagy modulators ${ }^{[27]}$.

We hope that the special issue presented here will attract attention and initiate extensive discussion of these new research directions including selective autophagy and cell death in neurons, eventually developing new translational targets and offering hope to the people who suffer greatly from these devastating neurodegenerative diseases. As the guest editor I thank all authors for their hard work in contributing to this special issue.

\section{REFERENCES}

[1] Lim J, Yue Z. Neuronal aggregates: formation, clearance, and spreading. Dev Cell 2015, 32: 491-501.

[2] Williams A, Jahreiss L, Sarkar S, Saiki S, Menzies FM, 
Ravikumar B, Rubinsztein DC. Aggregate-prone proteins are cleared from the cytosol by autophagy: therapeutic implications. Curr Top Dev Biol 2006, 76: 89-101.

[3] Feng Y, He D, Yao Z, Klionsky DJ. The machinery of macroautophagy. Cell Res 2014, 24: 24-41.

[4] Efeyan A, Comb WC, Sabatini DM. Nutrient-sensing mechanisms and pathways. Nature 2015, 517: 302-310.

[5] Kim J, Kundu M, Viollet B, Guan KL. AMPK and mTOR regulate autophagy through direct phosphorylation of Ulk1. Nat Cell Biol 2011, 13: 132-141.

[6] Stolz A, Ernst A, Dikic I. Cargo recognition and trafficking in selective autophagy. Nat Cell Biol 2014, 16: 495-501.

[7] Okamoto K. Organellophagy: eliminating cellular building blocks via selective autophagy. J Cell Biol 2014, 205: 435445.

[8] Rui YN, Xu Z, Patel B, Chen Z, Chen D, Tito A, et al. Huntingtin functions as a scaffold for selective macroautophagy. Nat Cell Biol 2015, 17: 262-275.

[9] Lippai M, Low P. The role of the selective adaptor p62 and ubiquitin-like proteins in autophagy. Biomed Res Int 2014, 2014: 832704

[10] Lim KL, Lim GG. K63-linked ubiquitination and neurodegeneration. Neurobiol Dis 2011, 43: 9-16.

[11] Mizumura K, Choi AM, Ryter SW. Emerging role of selective autophagy in human diseases. Front Pharmacol 2014, 5: 244.

[12] Lee MJ, Lee JH, Rubinsztein DC. Tau degradation: the ubiquitin-proteasome system versus the autophagy-lysosome system. Prog Neurobiol 2013, 105: 49-59.

[13] Polito VA, Li H, Martini-Stoica H, Wang B, Yang L, Xu Y, et al. Selective clearance of aberrant tau proteins and rescue of neurotoxicity by transcription factor EB. EMBO Mol Med 2014, 6: 1142-1160.

[14] Chen S, Zhang X, Song L, Le W. Autophagy dysregulation in amyotrophic lateral sclerosis. Brain Pathol 2012, 22: 110-116.

[15] Chen S, Zhang XJ, Li LX, Wang Y, Zhong RJ, Le W. Histone deacetylase 6 delays motor neuron degeneration by ameliorating the autophagic flux defect in a transgenic mouse model of amyotrophic lateral sclerosis. Neurosci Bull 2015, 31: 459-468.

[16] Zhang X, Chen S, Song L, Tang Y, Shen Y, Jia L, et al.
MTOR-independent, autophagic enhancer trehalose prolongs motor neuron survival and ameliorates the autophagic flux defect in a mouse model of amyotrophic lateral sclerosis. Autophagy 2014, 10: 588-602.

[17] Shen DN, Zhang LH, Wei EQ, Yang Y. Autophagy in synaptic development, function and pathology. Neurosci Bull 2015, 31: 416-426.

[18] Guo D, Ying Z, Wang $H$, Chen D, Gao F, Ren $H$, et al. Regulation of autophagic flux by CHIP. Neurosci Bull 2015, 31: 469-479.

[19] Cai Z, Zeng W, Tao K, E Z, Wang B, Yang Q. Chaperonemediated autophagy: roles in neuroprotection. Neurosci Bull 2015, 31: 452-458.

[20] Button RW, Luo S, Rubinsztein DC. Autophagy activity in neuronal cell death. Neurosci Bull 2015, 31: 382-394.

[21] Liu X, Huang S, Wang X, Li W, Mao Z. Chaperonemediated autophagy and neurodegeneration: connections, mechanisms, and therapeutic implications. Neurosci Bull 2015, 31: 407-415.

[22] Yuan $Y$, Zhang X, Zheng Y, Chen Z. Regulation of mitophagy in ischemic brain injury. Neurosci Bull 2015, 31: 395-406.

[23] Liang K, Zhu L, Tan J, Shi W, He Q, Yu B. Identification of autophagy signaling network that contributes to stroke in the ischemic rodent brain via gene expression. Neurosci Bull 2015, 31: 480-490.

[24] Liang P, Le W. The role of autophagy in the pathogenesis of multiple sclerosis. Neurosci Bull 2015, 31: 435-444.

[25] Wang HC, Zhang T, Kuerban B, Jin YL, Le W, Hara $\mathrm{H}$, et al. Autophagy is involved in oral $r A A V / A \beta$ vaccine-induced $A \beta$ clearance in APP/PS1 transgenic mice. Neurosci Bull 2015, 31: 491-504.

[26] Yang D, Zhu L, Ren J, Ma R, Zhu H, Xu J. Dysfunction of autophagy as the pathological mechanism of motor neuron disease based on a patient-specific disease model. Neurosci Bull 2015, 31: 445-451.

[27] Jia J, Le W. Molecular network of neuronal autophagy in the pathophysiology and treatment of depression. Neurosci Bull 2015, 31: 427-434.

[28] Li ZQ, Li LX, Mo N, Cao YY, Kuerban B, Liang YX, et al. Duration-dependent regulation of autophagy by isoflurane exposure in aged rats. Neurosci Bull 2015, 31: 505-513. 